\title{
German Heart Surgery Report 2015: The Annual Updated Registry of the German Society for Thoracic and Cardiovascular Surgery
}

\author{
Andreas Beckmann ${ }^{1}$ Anne-Katrin Funkat ${ }^{2}$ Jana Lewandowski ${ }^{1}$ Michael Frie ${ }^{3}$ Markus Ernst ${ }^{4}$ \\ Khosro Hekmat ${ }^{5}$ Wolfgang Schiller ${ }^{6}$ Jan F. Gummert ${ }^{7}$ Armin Welz ${ }^{6}$
}

${ }^{1}$ German Society for Thoracic and Cardiovascular Surgery, Langenbeck-Virchow-Haus, Berlin, Germany

${ }^{2}$ Leipzig Heart Institute, Leipzig, Germany

${ }^{3}$ FOM Hochschule für Oekonomie \& Management, Essen, Germany

${ }^{4}$ Department of Cardiac and Vascular Surgery, University Kiel, Kiel, Germany

${ }^{5}$ Department of Cardiothoracic Surgery, University of Cologne, Cologne, Germany

${ }^{6}$ Department of Cardiac Surgery, University Bonn, Bonn, Germany

${ }^{7}$ Department of Thoracic and Cardiovascular Surgery, Heart and

Diabetes Center NRW, Bad Oeynhausen, Germany

Thorac Cardiovasc Surg 2016;64:462-474.
Address for correspondence Andreas Beckmann, MD, German Society for Thoracic and Cardiovascular Surgery, Langenbeck-Virchow-Haus, Luisenstr 58/59, 10117 Berlin, Germany (e-mail: gf@dgthg.de).

\begin{abstract}
On the basis of a long-standing voluntary registry, which was founded by the German Society for Thoracic and Cardiovascular Surgery (GSTCVS), well-defined data of all heart, thoracic, and vascular surgery procedures performed in 78 German heart surgery departments during 2015 are analyzed. In 2015, a total of 103,967 heart surgery procedures (implantable cardioverter defibrillator, pacemaker, and extracardiac procedures without ECC excluded) were submitted to the database. Approximately $14.8 \%$ of the patients were at least 80 years old, resulting in an increase of $0.6 \%$ compared with the data of 2014 . For 38,601 isolated coronary artery bypass grafting procedures

Keywords

- heart surgery

- outcome

- registry

- quality assurance

- congenital heart disease

- aortic surgery

- transplantation (relationship on-/off-pump: 5:1), the unadjusted inhospital mortality was $2.7 \%$. Concerning the 32,346 isolated heart valve procedures (including 10,606 catheter-based implantations) an unadjusted inhospital mortality of $4.4 \%$ was observed.

This annual updated registry of the GSTCVS represents voluntary public reporting by accumulating actual information for nearly all heart surgical procedures in Germany, demonstrates advancements in heart medicine, and enables internal/external quality assurance for all participants. In addition, the registry demonstrates that the provision of heart surgery in Germany is appropriate and patients are treated nationwide in a round-the-clock service.
\end{abstract}

\section{Introduction}

Legitimate demands for a sophisticated quality management in medicine-by authorities, scientific organizations, health insurance companies, and patients all over the world-have stimulated quality awareness, resulting in the development of

received

August 9, 2016

accepted after revision

August 22, 2016

published online

September 1, 2016

versatile quality assurance activities such as benchmark projects, registries, and others to answer those needs. As early as 1978, the board of directors of the German Society for Thoracic and Cardiovascular Surgery (GSTCVS; www.dgthg. de) decided to set up an annual database of all cardiac surgical procedures in terms of a voluntary registry. Since 1989, the

(c) 2016 Georg Thieme Verlag KG Stuttgart · New York
DOI http://dx.doi.org/ 10.1055/s-0036-1592124. ISSN 0171-6425. 
data are annually updated, summarized in a registry format, and published in the scientific journal of the GSTCVS. ${ }^{1-26}$ The aims of this registry are to gather developments and current trends in cardiac surgery in Germany, to compile various results for nearly all cardiac surgical procedures, to enable each participating department for cardiac surgery comparing its own results to the nationwide achievements, and to facilitate an evaluation on an international level for the society.

In order to monitor actual conditions as well as developments in cardiac medicine, the registry covers all relevant techniques as well as innovative technologies including minimally invasive cardiac surgery and all kinds of heart valve operations including transcatheter heart valve interventions (e.g., transcatheter aortic valve implantation, TAVI). Thereby, important findings for current patient safety and the future of patient care are collected and may be evaluated under different aspects.

Data and results presented in this report comprehend assorted data of 2015.

\section{Material and Methods}

Since 2004, a standardized questionnaire gathers well-defined information for all individual procedures exactly described by an annually updated German adaption of the International Classification of Procedures in Medicine (ICPM) called operation code (Operationen- und Prozedurenschlüssel).

All participating institutions were requested to complete the structured questionnaire, asking for all performed procedures and associated inhospital mortality, by January 24 , 2016. The recommended path for data export is electronic transmission of an encrypted file to the office of the GSTCVS in Berlin. After transaction, the data are decrypted, evaluated for completeness, and compiled for further analysis, thus ensuring anonymity for each participating institution. This compilation algorithm guarantees a high compliance for submission of complete datasets.

Inclusion criteria for the 2015 registry were all cardiac surgical procedures performed on patients between January 1, 2015, and December 31, 2015, unrelated to the date of admission or discharge as compared with other registries. Like in all previous years, the number of procedures was counted rather than individual patients. For example, if during one admission a patient required coronary bypass grafting due to a complication following initial mitral valve reconstruction, one count in the "mitral valve reconstruction" category and another in the "coronary surgery" category are enumerated. Thus, the registry contains a higher number of procedures than the real number of patients operated on.

Death of patients was defined as inhospital mortality. Per definition, the observed mortality is always attributed to the first cardiac procedure, for example, the death of a patient requiring a replacement of the ascending aorta due to a complication of coronary artery bypass grafting (CABG) would only be attributed to the coronary procedure. The denominator in the calculation of mortality rates is getting falsly higher with the assignment of complication procedures resulting in a marginal underestimation of mortality rates (calculus estimate: $<0.1 \%$ ).

The main reason for this structural setup of the registry established over several decades is to keep in accordance with the German data privacy act with its specific regulations for patients. Furthermore, it seemed to be relevant getting not only the count of treated patients but also detailed information about all performed procedures. Last but not least, the process of data acquisition had to be standardized and feasible for all participating cardiac surgery departments in Germany, thus enabling the submission of a complete dataset, regardless of the locally existing hard- and software used for data management.

In 2015, a total of 78 institutions performed heart surgery in Germany. Fortunately, all units answered the questionnaire and delivered a complete dataset for 2015 including hospital mortality rates.

\section{Registry Data 2015}

-Table 1 gives an overview of heart surgical procedures by distribution between the 16 German states. In the table, the range of heart operations per 100,000 inhabitants shows a minimum of 101.8 (Baden-Württemberg) and a maximum of 168.9 (Sachsen-Anhalt), resulting in a nationwide mean value of 126.6. Analyzing the heart operations for department dimension, $59 \%$ performed from 590 to 1,460 procedures (-Table 2). Summarizing the departments by various heart surgical procedures, it can be ascertained that heart operations in patients for congenital heart disease $(<1$ year, with extracorporeal circulation [ECC]) are conducted in 23 and heart transplantations in 22 institutions ( - Table 3). - Table 4 illustrates the number of procedures using ECC from 2006 to 2015 in Germany. Over the past decade, the number of heart operations using ECC shows a diminution by 9,530 procedures, certainly an achievement of establishing innovations and minimally invasive therapies in heart surgery.

Overall, 185,270 procedures were reported to the registry in 2015, a decrease of $1.1 \%$ compared to $2014(187,392$ procedures). A total of 103,967 heart surgical procedures (excluding implantable cardioverter defibrillator, pacemakers, and miscellaneous procedures without ECC) in 2015 displays nearly the same quantity with a difference of just $0.5 \% \quad(n=517) \quad$ compared to $2014 \quad(104,484$ procedures; - Table 5). - Tables 6 and 7, V1 to V7, C1 to C2, -Table Con1 and Con2, and -Table Mis 1 to Mis 5 and -Figs. 1-9 demonstrate some compiled registry data of 2015 under different aspects and for various categories.

Compared to previous years' data, several important developments continued to be almost unchanged in 2015. The

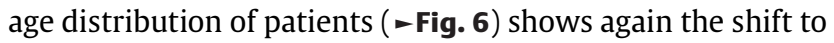
an elderly patient population, with presently $53.1 \%$ of the cardiac procedures performed in patients aged at least 70 years and $14.8 \%$ in patients aged 80 years or older. However, mortality remained on the same low level over the represented decade (-Fig. $\mathbf{2}$ ). The rate of CABG procedures decreased over the past years, whereas the relative 
number of off-pump CABG procedures reached a level of $16.5 \%$ in 2015 as compared to $15.3 \%$ in 2014 (-Fig. 3).

Since 2004 , more than $50 \%$ of isolated mitral valve procedures have been reconstructions. In 2015, mitral valve reconstruction could be achieved in $63.6 \%$ of the procedures (-Fig. 8). Based on the fact that without exception all isolated mitral valve procedures are included, regardless of the underlying valve disease concerning morphology or urgency of operation, it has to be assumed that the relative rate of mitral valve reconstruction would certainly be even higher if patients without possibility or indication for reconstruction would have been excluded (e.g., mitral valve stenosis, calcifications, or endocarditis). In other publications, for example by Gammie et al, ${ }^{27}$ patients with mitral valve stenosis, endocarditis, and emergency procedures were usually excluded. Therefore, the published rates of mitral valve repair have to be interpreted with caution if compared with this registry.

The continued increase of left ventricular assist device implantations (-Fig. 10) emphasizes the increasing relevance of mechanical circulatory support.

Again, a remarkable trend is the still ongoing increase of TAVI procedures in Germany (-Fig. 5), whereas the count of isolated aortic valve replacement procedures showed a slight decrease of $4.9 \%$. Starting in 2006 with just 78 implantations ( $0.67 \%$ of isolated aortic valve procedures), in $2015,9,813$ (46.7\%) TAVI were reported to the registry. It must be emphasized that the 78 institutions which contribute their data to this registry do not represent all departments performing TAVI in Germany. It is known that some additional institutions in Germany perform TAVI procedures under different infrastructure conditions and various external cooperations. This practice does not correlate to the recommendations of the European guideline on the management of valvular heart disease (version 2012). ${ }^{28}$ In July 2015, the first version of the quality assurance directive for "minimally invasive heart valve interventions (TAVI, mitral clip reconstruction)," in which obligatory structures, defined processes, and qualified personnel are exactly specified, was released by the German Federal Joint Committee (G-BA).

In addition to the registry data, the short-, mid-, and longterm results of the German Aortic Valve Registry (GARY) ${ }^{29-33}$ and the annual analyses of the legal quality assurance ( $\$ 137$ SGB V) are of outstanding importance concerning the judgement of developments and quality - and thus for patient benefit.

\section{Discussion}

The registry of the GSTCVS enables a comprehensive overview of all heart surgical procedures performed in Germany in 2015. The accuracy of this registry is considered to be high due to the implemented compilation algorithm using standardized operation coding which is a relevant criterion for reimbursement purposes. This is supported by other authors who could demonstrate a high accuracy for major outcome parameters in unaudited registries. ${ }^{34}$ In continuation with previous years, it can be concluded that heart surgery in Germany is performed on a constantly high level with very high inhospital survival rates of patients compared with international registries. In addition, the registry data demonstrate that the provision of heart surgery in Germany is appropriate and nationwide patient treatment is guaranteed in a round-the-clock service (24/7/365). These conclusions are especially important in the context of various activities in health care policy and the demographic change of the German population leading to an increase of patient age and related comorbidities resulting in higher preoperative risk profiles.

Compared to 2014, the number of cardiac surgery procedures remains on nearly the same level due to the still increasing number of catheter-based heart valve procedures.

Further improvements in the basic configuration of the registry are recommended to enable more detailed and particularly risk-adjusted data analyses. However, if significant structural changes in data collection for the registry are conducted, it must be ensured that data compatibility still allows further longitudinal data analysis.

Completeness, validity, and further developments will depend on continued efforts of the GSTCVS in close collaboration with all cardiac surgical departments in Germany. This will be of outstanding importance in the sense of a contribution for patient safety and to adduce evidence for high quality of heart surgery in Germany.

\section{Abbreviations}

ASD atrial septal defect

CABG coronary artery bypass grafting

CHD congenital heart disease

DLTx double lung transplantation

DORV double outlet right ventricle

ECC extracorporeal circulation

HLTx heart-lung transplantation

HTx heart transplantation

ICD implantable cardioverter defibrillator

PDA patent ductus arteriosus

SLTx single lung transplantation

TAVI transcatheter aortic valve implantation

TGA transposition of the great arteries

TMLR transmyocardial laser revascularisation

Tx Organ transplantation

VSD ventricular septal defect
Acknowledgments

On behalf of the German Society for Thoracic and Cardiovascular Surgery, the authors would like to thank the heads of the departments and their employees of all cardiac surgery units in Germany for their continued cooperation and support to realize this registry. 


\section{Tables and Figures}

Table 1 Heart operations in German states

\begin{tabular}{|l|l|l|l|}
\hline German states & Quantity $^{\text {a }}$ & Population $^{\text {b }}$ & $\begin{array}{l}\text { Heart procedures/ } \\
100,000 \\
\text { inhabitants }\end{array}$ \\
\hline Baden-Württemberg & 10,907 & $10,716,644$ & 101.8 \\
\hline Bayern & 14,542 & $12,691,568$ & 114.6 \\
\hline Berlin & 3,800 & $3,469,849$ & 109.5 \\
\hline Brandenburg & 3,194 & $2,457,872$ & 129.9 \\
\hline Bremen & 812 & 661,888 & 122.7 \\
\hline Hamburg & 2,168 & $1,762,791$ & 123.0 \\
\hline Hessen & 7,875 & $6,093,888$ & 129.2 \\
\hline Mecklenburg-Vorpommern & 2,134 & $1,599,138$ & 133.4 \\
\hline Niedersachsen & 10,848 & $7,826,739$ & 138.6 \\
\hline Nordrhein-Westfalen & 23,164 & $17,638,098$ & 131.3 \\
\hline Rheinland-Pfalz & 5,637 & $4,011,582$ & 140.5 \\
\hline Saarland & 1,644 & 989,035 & 166.2 \\
\hline Sachsen & 5,271 & $4,055,274$ & 130.0 \\
\hline Sachsen-Anhalt & 3,776 & $2,235,548$ & 168.9 \\
\hline Schleswig-Holstein & 4,180 & $2,830,864$ & 147.7 \\
\hline Thüringen & 2,843 & $2,156,759$ & 131.8 \\
\hline Total & 102,795 & $81,197,537$ & 126.6 \\
\hline
\end{tabular}

${ }^{\mathrm{a}} 1,172$ foreign residents excluded.

${ }^{b}$ Federal Offices for Statistics of German Population; due date December 31, 2014.

Table 2 Departments sorted by quantity $(n=103.967)$

\begin{tabular}{|l|l|l|l|l|l|}
\hline $\begin{array}{l}\text { Procedures } \\
\text { (quantity) }\end{array}$ & $<\mathbf{5 0 0}$ & $\begin{array}{l}\mathbf{5 0 0 -} \\
\mathbf{9 9 9}\end{array}$ & $\begin{array}{l}\mathbf{1 , 0 0 0 -} \\
\mathbf{1 , 4 9 9}\end{array}$ & $\begin{array}{l}\mathbf{1 , 5 0 0 -} \\
\mathbf{1 , 9 9 9}\end{array}$ & $\begin{array}{l}\mathbf{2 , 0 0 0 -} \\
\mathbf{5 , 0 0 0}\end{array}$ \\
\hline $\begin{array}{l}\text { Departments } \\
\text { (no.) }\end{array}$ & 7 & 21 & 25 & 12 & 13 \\
\hline Average & 346 & 814 & 1,198 & 1,663 & 2,658 \\
\hline Range & $\begin{array}{l}233- \\
445\end{array}$ & $\begin{array}{l}590- \\
992\end{array}$ & $\begin{array}{l}1,032- \\
1,460\end{array}$ & $\begin{array}{l}1,508- \\
1,966\end{array}$ & $\begin{array}{l}2,021- \\
4,102\end{array}$ \\
\hline
\end{tabular}

Note: Pacemaker/ implantable cardioverter defibrillator and extracardiac surgery without extracorporeal circulation excluded.
Table 3 Departments summarized by heart surgery procedures in 2015

\begin{tabular}{|l|l|}
\hline Procedures & $N$ \\
\hline Coronary artery bypass grafting & 77 \\
\hline Heart valve surgery & 77 \\
\hline Surgery for CHD patients $<1$ yr with ECC & $23^{\text {a }}$ \\
\hline HTx & $22^{\mathrm{b}}$ \\
\hline HLTx & 2 \\
\hline
\end{tabular}

${ }^{\mathrm{a}} \mathrm{N}=2,041: 3-17$ procedures in 4 units, $22-46$ procedures in 5 units, $65-89$ procedures in 5 units, and $110-265$ procedures in 9 units.

${ }^{\mathrm{b}} \mathrm{N}=283: 23-75 \mathrm{Tx}$ in 4 units, $11-17 \mathrm{Tx}$ in 5 units, $5-8 \mathrm{Tx}$ in 8 units, and 2-4 Tx in 5 units.

Table 4 Open heart procedures using extracorporeal circulation (2006-2015)

\begin{tabular}{|l|l|l|l|l|l|l|l|l|l|l|}
\hline & $\mathbf{2 0 0 6}$ & $\mathbf{2 0 0 7}$ & $\mathbf{2 0 0 8}$ & $\mathbf{2 0 0 9}$ & $\mathbf{2 0 1 0}$ & $\mathbf{2 0 1 1}$ & $\mathbf{2 0 1 2}$ & $\mathbf{2 0 1 3}$ & $\mathbf{2 0 1 4}$ & $\mathbf{2 0 1 5}$ \\
\hline Departments & 80 & 80 & 79 & 80 & 79 & 78 & 79 & 79 & 78 & 78 \\
\hline Procedures & 91,057 & 91,618 & 89,773 & 86,916 & 84,686 & 84,402 & 84,388 & 84,040 & 83,787 & 81,527 \\
\hline Average & 1,138 & 1,145 & 1,136 & 1,086 & 1,072 & 1,082 & 1,068 & 1,064 & 1,074 & 1,045 \\
\hline
\end{tabular}


Table 5 Heart surgery categories 2015

\begin{tabular}{|l|l|l|l|l|}
\hline Category & with ECC & without ECC & Total & Diff. 2014 (\%) \\
\hline Coronary artery bypass grafting & 45,107 & 6,834 & 51,941 & -3.5 \\
\hline Heart valve procedures & 21,892 & 10,454 & 32,346 & +3.1 \\
\hline Surgery of thoracic aorta & 7,265 & 657 & 7,922 & +2.2 \\
\hline Surgery for CHD & 4,913 & 979 & 5,892 & +2.0 \\
\hline Cardiac surgery and others & 1,173 & 1,206 & 2,379 & -3.8 \\
\hline Assist device procedures & 765 & 2,310 & 3,075 & +5.7 \\
\hline Extracardiac surgery & 358 & 54,785 & 55,143 & -3.4 \\
\hline Pacemaker and ICD procedures & 54 & 26,518 & 26,572 & +1.2 \\
\hline Total & 81,527 & 103,743 & 185,270 & -1.1 \\
\hline
\end{tabular}

Table 6 Additional data for heart surgery procedures with ECC in 2015 versus 2014

\begin{tabular}{|l|l|l|l|l|}
\hline \multirow{2}{*}{ Procedures } & \multicolumn{2}{|l|}{2015} & 2014 \\
\cline { 2 - 5 } & $\boldsymbol{N}$ & $\%$ & $\mathrm{~N}$ & $\%$ \\
\hline Emergency & 12,843 & $12.4 \%$ & 12,583 & $12.0 \%$ \\
\hline Redo & 8,994 & $8.7 \%$ & 8,809 & $8.4 \%$ \\
\hline
\end{tabular}

Table 7 Gender distribution

\begin{tabular}{|l|l|l|}
\hline & Female (\%) & Male (\%) \\
\hline Heart valve procedures & 44 & 56 \\
\hline Coronary procedures & 22 & 78 \\
\hline Surgery for CHD & 44 & 56 \\
\hline Surgery of thoracic aorta & 33 & 67 \\
\hline Cardiac surgery, other & 52 & 48 \\
\hline Assist Devices & 29 & 71 \\
\hline Pacemaker and ICD & 35 & 65 \\
\hline Extracardiac surgery & 34 & 66 \\
\hline Total & 33 & 67 \\
\hline
\end{tabular}

Table V1 Isolated heart valve procedures

\begin{tabular}{|l|l|l|l|}
\hline Procedures & $N$ & $\dagger$ & $\%$ \\
\hline Single & 17,986 & 649 & 3.6 \\
\hline Double & 3,280 & 305 & 9.3 \\
\hline Triple & 358 & 47 & 13.1 \\
\hline Transcatheter access(single valve) & 10,581 & 405 & 3.8 \\
\hline Transcatheter access(double valve) & 25 & 2 & 8.0 \\
\hline Not specified & 116 & 6 & 5.2 \\
\hline Total & 32,346 & 1,414 & 4.4 \\
\hline
\end{tabular}

Transcatheter heart valve procedures: 9,813 aortic valve implantations; 111 mitral valve implantations; 632 mitral valve repairs; 1 tricuspid valve implantation; 24 tricuspidal valve repairs; 25 combined aortic and mitral valve procedures; no pulmonary valve implantation.
Table V2 Single heart valve procedures: access type

\begin{tabular}{|l|l|l|l|}
\hline Heart valve/access & $N$ & $\dagger$ & $\%$ \\
\hline Aortic valve & 21,120 & 715 & 3.4 \\
\hline Sternotomy & 8,291 & 292 & 3.5 \\
\hline Part. sternotomy & 3,016 & 49 & 1.6 \\
\hline Transvascular & 7,311 & 218 & 3.0 \\
\hline Transapical & 2,502 & 156 & 6.2 \\
\hline Mitral valve & 6,770 & 283 & 4.2 \\
\hline Sternotomy & 3,099 & 205 & 6.6 \\
\hline Minimally invasive & 2,928 & 49 & 1.7 \\
\hline Transcatheter & 743 & 29 & 3.9 \\
\hline Tricuspidal valve & 617 & 53 & 8.6 \\
\hline Sternotomy & 444 & 45 & 10.1 \\
\hline Minimally invasive & 148 & 6 & 4.1 \\
\hline Transcatheter & 25 & 2 & 8.0 \\
\hline Pulmonary valve & 56 & 3 & 5.4 \\
\hline Sternotomy & 56 & 3 & 5.4 \\
\hline Minimally invasive & 0 & 0 & - \\
\hline Transcatheter & 0 & 0 & - \\
\hline Total & 28,563 & 1,054 & 3.7 \\
\hline
\end{tabular}

Note: Apical aortic conduits procedures $(n=4)$ excluded.

Table V3 Isolated aortic valve procedures

\begin{tabular}{|l|l|l|l|}
\hline Prosthesis/repair & $\boldsymbol{N}$ & $\dagger$ & $\%$ \\
\hline Xenograft & 9,839 & 311 & 3.2 \\
\hline Mechanical prosthesis & 1,311 & 23 & 1.8 \\
\hline Repair & 124 & 5 & 4.0 \\
\hline Homograft & 33 & 2 & 6.1 \\
\hline Total & 11,307 & 341 & 3.0 \\
\hline
\end{tabular}

Table V4 Isolated mitral valve procedures

\begin{tabular}{|l|l|l|l|}
\hline Prosthesis/repair & $N$ & $\dagger$ & $\%$ \\
\hline Repair & 3,833 & 52 & 1.4 \\
\hline Xenograft & 1,680 & 165 & 9.8 \\
\hline Mechanical prosthesis & 505 & 35 & 6.9 \\
\hline Homograft & 9 & 2 & 22.2 \\
\hline Total & 6,027 & 254 & 4.2 \\
\hline
\end{tabular}


Table V5 Multiple heart valve procedures

\begin{tabular}{|l|l|l|l|}
\hline & $N$ & $\dagger$ & $\%$ \\
\hline Mitral + tricuspid & 1,685 & 118 & 7.0 \\
\hline Aortic + mitral & 1,372 & 170 & 12.4 \\
\hline Aortic + mitral + tricuspid & 357 & 47 & 13.2 \\
\hline Aortic + tricuspid & 174 & 15 & 8.6 \\
\hline Aortic + pulmonary ${ }^{\mathrm{a}}$ & 39 & 1 & 2.6 \\
\hline Tricuspid + pulmonary & 10 & 1 & 10.0 \\
\hline Aortic + mitral + pulmonary & 1 & 0 & 0.0 \\
\hline Total & 3,638 & 352 & 9.7 \\
\hline
\end{tabular}

Note: Transcatheter procedures excluded.

${ }^{\mathrm{a}}$ Ross procedures included.

Table V6 Isolated/combined mitral valve procedures: implantation/replacement vs. repair

\begin{tabular}{|c|l|l|l|l|l|l|l|l|l|l|}
\hline Mitral valve surgery & \multicolumn{3}{l|}{ Repair } & \multicolumn{3}{l|}{$\begin{array}{l}\text { Implantation/replace- } \\
\text { ment }\end{array}$} & \multicolumn{3}{l|}{ All procedures } \\
\hline & $N$ & $\dagger$ & $\%$ & $N$ & $\dagger$ & $\%$ & $N$ & $\%$ repair & $\dagger$ & $\%$ \\
\hline Isolated & 3,833 & 52 & 1.4 & 2,194 & 202 & 9.2 & 6,027 & 63.6 & 254 & 4.2 \\
\hline+ Aortic valve & 603 & 38 & 6.3 & 769 & 132 & 17.2 & 1,372 & 44.0 & 170 & 12.4 \\
\hline+ Tricuspid valve repair ${ }^{\text {a }}$ & 1,025 & 44 & 4.3 & 611 & 66 & 10.8 & 1,636 & 62.7 & 110 & 6.7 \\
\hline+ CABG & 1,711 & 132 & 7.7 & 918 & 134 & 14.6 & 2,629 & 65.1 & 266 & 10.1 \\
\hline+ CABG + aortic valve replacement & 318 & 40 & 12.6 & 286 & 59 & 20.6 & 604 & 52.6 & 99 & 16.4 \\
\hline Total & 7,490 & 306 & 4.1 & 4,778 & 593 & 12.4 & 12.268 & 61.1 & 899 & 7.3 \\
\hline
\end{tabular}

${ }^{\mathrm{a}} \mathrm{A}$ total of 49 procedures (not specified mitral valve + tricuspid valve surgery) excluded; $\uparrow:$ (8/49) $16 \%$.

Table V7 Transcatheter heart valve procedures

\begin{tabular}{|c|c|c|c|c|c|c|c|}
\hline & \multicolumn{2}{|c|}{ without ECC } & \multicolumn{2}{|c|}{ with ECC } & \multicolumn{3}{|c|}{ All procedures } \\
\hline & $N$ & $\dagger$ & $N$ & $\dagger$ & $N$ & $\dagger$ & $\%$ \\
\hline Aortic valve implantation & 9,675 & 330 & 138 & 44 & 9,813 & 374 & 4 \\
\hline Transvascular & 7,236 & 192 & 75 & 26 & 7,311 & 218 & 3 \\
\hline Transapical & 2,439 & 138 & 63 & 18 & 2,502 & 156 & 6 \\
\hline Mitral valve procedure & 731 & 26 & 12 & 3 & 743 & 29 & 4 \\
\hline Repair & 625 & 20 & 7 & 1 & 632 & 21 & 3 \\
\hline Implantation & 106 & 6 & 5 & 2 & 111 & 8 & 7 \\
\hline Tricuspidal valve procedure & 23 & 1 & 2 & 1 & 25 & 2 & 8 \\
\hline Repair & 22 & 1 & 2 & 1 & 24 & 2 & 8 \\
\hline Implantation & 1 & 0 & 0 & 0 & 1 & 0 & 0 \\
\hline Aortic + mitral valve implantation & 25 & 2 & 0 & 0 & 25 & 2 & 8 \\
\hline Aortic valve implantation ${ }^{\mathrm{a}}+\mathrm{CABC}$ & 37 & 2 & 13 & 8 & 50 & 10 & 20 \\
\hline Mitral valve implantation ${ }^{\mathrm{b}}+\mathrm{CABG}$ & 1 & 0 & 1 & 1 & 2 & 1 & 50 \\
\hline Aortic + mitral valve + CABG & 2 & 0 & 0 & 0 & 2 & 0 & 0 \\
\hline Total & 10,494 & 361 & 166 & 57 & 10,660 & 418 & 4 \\
\hline
\end{tabular}

Note: Pulmonary valve implantations for the correction of congenital lesions are not included; No procedure was reported for adults without congenital lesion. $25 \%$ of TAVI by transapical access; $1.5 \%$ of TAVI under use of ECC.

${ }^{\mathrm{a}}$ Femoral, subclavian, or transaortic access.

bTransvascular and transapical access. 
Table C1 Isolated/combined CABG with ECC

\begin{tabular}{|l|l|l|l|}
\hline & $N$ & $\dagger$ & $\%$ \\
\hline Isolated CABG & 38,601 & 1,057 & 2.7 \\
\hline+ Aortic valve replacement & 7,214 & 396 & 5.5 \\
\hline+ Other & 2,705 & 156 & 5.8 \\
\hline+ Mitral valve repair & 1,711 & 132 & 7.7 \\
\hline+ Mitral valve replacement & 918 & 134 & 14.6 \\
\hline + Aortic valve replacement + mitral valve repair & 318 & 40 & 12.6 \\
\hline + Aortic + mitral valve replacement & 286 & 59 & 20.6 \\
\hline+ Aneurysm resection & 133 & 7 & 5.3 \\
\hline+ Transcatheter aortic valve implantation & 50 & 10 & 20.0 \\
\hline+ TMLR & 1 & 0 & 0.0 \\
\hline Total & 51,937 & 1,991 & 3.8 \\
\hline
\end{tabular}

Table C2 Isolated CABG with/without ECC

\begin{tabular}{|l|l|l|l|l|l|l|l|l|l|}
\hline Grafts $(N)$ & \multicolumn{2}{l}{ with ECC } & \multicolumn{2}{l|}{ without ECC } & \multicolumn{4}{l|}{ All } \\
\hline & $N$ & $\dagger$ & $\%$ & $N$ & $\dagger$ & $\%$ & $N$ & & $\dagger$ \\
\hline Single & 1,009 & 56 & 5.6 & 1,359 & 32 & 2.4 & 2,368 & 88 & 3.7 \\
\hline Double & 6,840 & 236 & 3.5 & 1,755 & 33 & 1.9 & 8,595 & 269 & 3.1 \\
\hline Triple & 14,353 & 388 & 2.7 & 2,185 & 38 & 1.7 & 16,538 & 426 & 2.6 \\
\hline Quadruple & 7,776 & 206 & 2.6 & 847 & 7 & 0.8 & 8,623 & 213 & 2.5 \\
\hline Quintuple + more & 2,273 & 60 & 2.6 & 204 & 1 & 0.5 & 2,477 & 61 & 2.5 \\
\hline Total & 32,251 & 946 & 2.9 & 6,350 & 111 & 1.7 & 38,601 & 1,057 & 2.7 \\
\hline
\end{tabular}

Table Con1 Congenital heart surgery with/without ECC

\begin{tabular}{|l|l|l|l|l|l|l|}
\hline \multirow{2}{*}{ Age (yrs) } & \multicolumn{2}{|l|}{$N$} & $\dagger$ & \multicolumn{3}{l|}{$\%$} \\
\cline { 2 - 8 } & with ECC & without ECC & with ECC & without ECC & with ECC & without ECC \\
\hline$<1$ & 2,041 & 736 & 63 & 13 & 3.1 & 1.8 \\
\hline $1-17$ & 1,796 & 206 & 9 & 1 & 0.5 & 0.5 \\
\hline$\geq 18$ & 1,076 & 37 & 24 & 1 & 2.2 & 2.7 \\
\hline Total & 4,913 & 979 & 96 & 15 & 2.0 & 1.5 \\
\hline
\end{tabular}

Table Con2 Lesions/procedures for CHD with and without ECC

\begin{tabular}{|l|l|l|l|l|l|l|l|l|l|}
\hline \multirow{2}{*}{ Lesion/procedures } & \multicolumn{2}{l|}{ Age $<\mathbf{1}$ yr } & \multicolumn{3}{l|}{ Age 1-17 yrs } & \multicolumn{2}{l|}{ Age $\geq 18$ yrs } \\
\cline { 2 - 11 } & $N$ & $\dagger$ & $\%$ & $N$ & $\dagger$ & $\%$ & $N$ & $\dagger$ & $\%$ \\
\hline ASD & 33 & 0 & 0.0 & 254 & 1 & 0.4 & 246 & 4 & 1.6 \\
\hline Complete AV canal & 183 & 2 & 1.1 & 88 & 1 & 1.1 & 10 & 1 & 10.0 \\
\hline VSD & 305 & 0 & 0.0 & 86 & 0 & 0.0 & 25 & 2 & 8.0 \\
\hline Fallot's tetralogy & 205 & 3 & 1.5 & 49 & 0 & 0.0 & 2 & 0 & 0.0 \\
\hline DORV & 48 & 0 & 0.0 & 21 & 1 & 4.8 & 1 & 0 & 0.0 \\
\hline TGA & 160 & 2 & 1.3 & 10 & 0 & 0.0 & 2 & 0 & 0.0 \\
\hline TGA + VSD & 63 & 2 & 3.2 & 10 & 0 & 0.0 & 0 & 0 & - \\
\hline
\end{tabular}


Table Con2 (Continued)

\begin{tabular}{|c|c|c|c|c|c|c|c|c|c|}
\hline \multirow[t]{2}{*}{ Lesion/procedures } & \multicolumn{3}{|c|}{ Age $<1 \mathrm{yr}$} & \multicolumn{3}{|c|}{ Age $1-17$ yrs } & \multicolumn{3}{|c|}{ Age $\geq 18$ yrs } \\
\hline & $N$ & $\dagger$ & $\%$ & $N$ & $\dagger$ & $\%$ & $N$ & $\bar{\dagger}$ & $\%$ \\
\hline Truncus arteriosus & 38 & 3 & 7.9 & 7 & 0 & 0.0 & 0 & 0 & - \\
\hline Fontan & 1 & 0 & 0.0 & 253 & 3 & 1.2 & 6 & 1 & 16.7 \\
\hline Norwood & 146 & 20 & 13.7 & 1 & 0 & 0.0 & 0 & 0 & - \\
\hline Pulmonary valve & 73 & 2 & 2.7 & 240 & 1 & 0.4 & 75 & 0 & 0.0 \\
\hline Transcatheter pulmonary valve implantation & 0 & - & - & 4 & 0 & 0.0 & 17 & 0 & 0.0 \\
\hline Aortic valve & 61 & 2 & 3.3 & 246 & 0 & 0.0 & 394 & 5 & 1.3 \\
\hline Ross procedure & 7 & 1 & 14.3 & 21 & 0 & 0.0 & 29 & 0 & 0.0 \\
\hline Mitral valve & 46 & 4 & 8.7 & 88 & 0 & 0.0 & 108 & 8 & 7.4 \\
\hline Tricuspid valve & 95 & 1 & 1.1 & 59 & 0 & 0.0 & 62 & 2 & 3.2 \\
\hline PDA & 225 & 3 & 1.3 & 26 & 1 & 3.8 & 2 & 0 & 0.0 \\
\hline Coarctation & 218 & 4 & 1.8 & 39 & 0 & 0.0 & 7 & 0 & 0.0 \\
\hline Others & 890 & 28 & 3.1 & 479 & 3 & 0.6 & 119 & 2 & 1.7 \\
\hline HTX & 7 & 0 & 0.0 & 42 & 0 & 0.0 & 0 & 0 & - \\
\hline HLTX & 0 & - & - & 0 & 0 & - & 0 & 0 & - \\
\hline LTx & 0 & - & - & 9 & 0 & 0.0 & 0 & 0 & - \\
\hline Total & 2,804 & 77 & 2.7 & 2,032 & 11 & 0.5 & 1,105 & 25 & 2.3 \\
\hline
\end{tabular}

Table Mis1 Ross procedures (autologous AV- and PVR)

\begin{tabular}{|l|l|l|l|l|l|l|l|l|l|l|}
\hline Age (y) & 2006 & 2007 & 2008 & 2009 & 2010 & 2011 & 2012 & 2013 & 2014 & 2015 \\
\hline$<18$ & 50 & 34 & 42 & 54 & 43 & 40 & 36 & 33 & 37 & 28 \\
\hline$\geq 18$ & 228 & 261 & 207 & 175 & 184 & 134 & 117 & 107 & 90 & 64 \\
\hline Total & 278 & 295 & 249 & 229 & 227 & 174 & 153 & 140 & 127 & 92 \\
\hline
\end{tabular}

Table Mis2 Heart and lung transplantation

\begin{tabular}{|c|c|c|c|c|c|c|}
\hline & \multicolumn{3}{|c|}{ with ECC } & \multicolumn{3}{|c|}{ without ECC } \\
\hline & $N$ & $\dagger$ & $\%$ & $N$ & $\dagger$ & $\%$ \\
\hline $\mathrm{HTx}$ & 283 & 38 & 13,4 & & & \\
\hline HLTX & 2 & 0 & 0,0 & & & \\
\hline LTx & 57 & 13 & 22,8 & 206 & 13 & 6,3 \\
\hline
\end{tabular}

Eurotransplant (ET) report for 2015: 283 HTx, 1 HTx + kidneyTx, 0 HTx + liverTx, 2 HLTx, 262 DLTx, 27 SLTx, 0 LTx + kidneyTx and 4 LTx + liverTx.

Table Mis3 Aortic surgery

\begin{tabular}{|l|l|l|l|l|l|l|}
\hline Replacement $^{\text {a }}$ & \multicolumn{2}{|l|}{ with ECC } & \multicolumn{2}{l|}{ without ECC } \\
\hline & $N$ & $\dagger$ & $\%$ & $N$ & $\dagger$ & \% \\
\hline Supracoronary replacement of ascending aorta & 1,433 & 127 & 8.9 & & & \\
\hline Supracoronary ascending + aortic valve replacement & 1,329 & 63 & 4.7 & & & \\
\hline Infracoronary ascending & & & - & & & \\
\hline Mechanical valve conduits & 508 & 36 & 7.1 & & & \\
\hline Biological valve conduits & 989 & 109 & 11.0 & & & \\
\hline David procedure & 514 & 8 & 1.6 & & & \\
\hline
\end{tabular}


Table Mis3 (Continued)

\begin{tabular}{|c|c|c|c|c|c|c|}
\hline \multirow[t]{2}{*}{ Replacement $^{\mathrm{a}}$} & \multicolumn{3}{|c|}{ with ECC } & \multicolumn{3}{|c|}{ without ECC } \\
\hline & $N$ & $\dagger$ & $\%$ & $N$ & $\dagger$ & $\%$ \\
\hline Yacoub procedure & 89 & 3 & 3.4 & & & \\
\hline Other & 279 & 23 & 8.2 & & & \\
\hline Aortic arch replacement $^{\mathrm{b}}$ & 1,977 & 271 & 13.7 & & & \\
\hline Replacement of descending aorta & 56 & 4 & 7.1 & 8 & 0 & 0.0 \\
\hline Thoracoabdominal aortic replacement & 86 & 10 & 11.6 & 24 & 4 & 16.7 \\
\hline Endostent descending aorta & 5 & 0 & 0.0 & 625 & 53 & 8.5 \\
\hline Total & 7,265 & 654 & 9.0 & 657 & 57 & 8.7 \\
\hline
\end{tabular}

aprocedures for abdominal aortic diseases excluded: 549 abdominal procedures and 609 endovascular abdominal stents.

${ }^{\mathrm{b}}$ All possible combined procedures included; the only common denominator is aortic arch surgery.

Table Mis4 Pacemaker and ICD procedures

\begin{tabular}{|l|l|l|l|l|l|l|l|}
\hline \multirow{2}{*}{ Device/category } & $N$ & $\dagger$ & $\%$ & \multicolumn{2}{l|}{ with ECC } & \multicolumn{2}{l|}{ without ECC } \\
\cline { 2 - 8 } & & & & $N$ & $\dagger$ & $N$ & $\dagger$ \\
\hline Pacemaker & 14,295 & 98 & 0.7 & 27 & 1 & 14,268 & 97 \\
\hline Implantation & 9,337 & 68 & 0.7 & 8 & 1 & 9,329 & 67 \\
\hline Battery exchange & 1,935 & 0 & 0.0 & 0 & 0 & 1,935 & 0 \\
\hline Revision & 3,023 & 30 & 1.0 & 19 & 0 & 3,004 & 30 \\
\hline ICD & 10,104 & 83 & 0.8 & 26 & 5 & 10,078 & 78 \\
\hline Implantation & 4,673 & 19 & 0.4 & 0 & 0 & 4,673 & 19 \\
\hline Battery exchange & 2,048 & 3 & 0.1 & 0 & 0 & 2,048 & 3 \\
\hline Revision & 3,383 & 61 & 1.8 & 26 & 5 & 3,357 & 56 \\
\hline Miscellaneous & 2,173 & 13 & 0.6 & 1 & 0 & 2,172 & 13 \\
\hline Total & 26,572 & 194 & 0.7 & 54 & 6 & 26,518 & 188 \\
\hline
\end{tabular}

Table Mis5 Surgical ablation therapy

\begin{tabular}{|l|l|l|l|}
\hline Energy source & \multirow{2}{*}{ Total } & Endocardiac & Epicardiac \\
\cline { 3 - 4 } & & $N$ & $N$ \\
\hline Unipolar radiofrequency & 229 & 77 & 152 \\
\hline Unipolar cryoradiofrequency & 254 & 122 & 132 \\
\hline Bipolar radiofrequency & 2.306 & 309 & 1,997 \\
\hline Cryothermy & 1.606 & 1,340 & 266 \\
\hline Microwave & 13 & 3 & 10 \\
\hline Focused ultrasound & 66 & 7 & 59 \\
\hline Laser & 9 & 1 & 8 \\
\hline Other & 23 & 4 & 19 \\
\hline Total & 4,506 & 1,863 & 2,643 \\
\hline
\end{tabular}

Note: 496 procedures unspecified with regard to endocardiac/epicardiac ablation. 


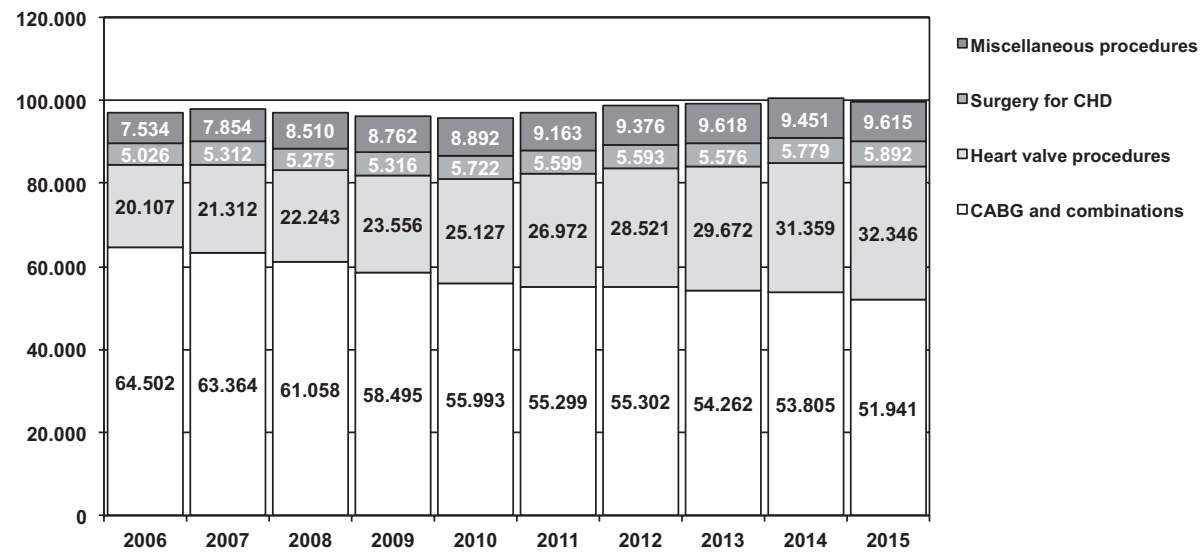

Fig. 1 Cardiac surgery in Germany (2006-2015). (1) Coronary artery bypass grafting (CABG) and combined procedures: all types of coronary surgery. (2) Heart valve procedures: combination with aortic surgery summarized under miscellaneous procedures. (3) Congenital heart surgery: atrial septal defect repair in adults or in combination with CABG or heart valve procedures are summarized in the CABG or heart valve procedure groups. (4) Miscellaneous procedures: all other types of procedures with extracorporeal circulation.

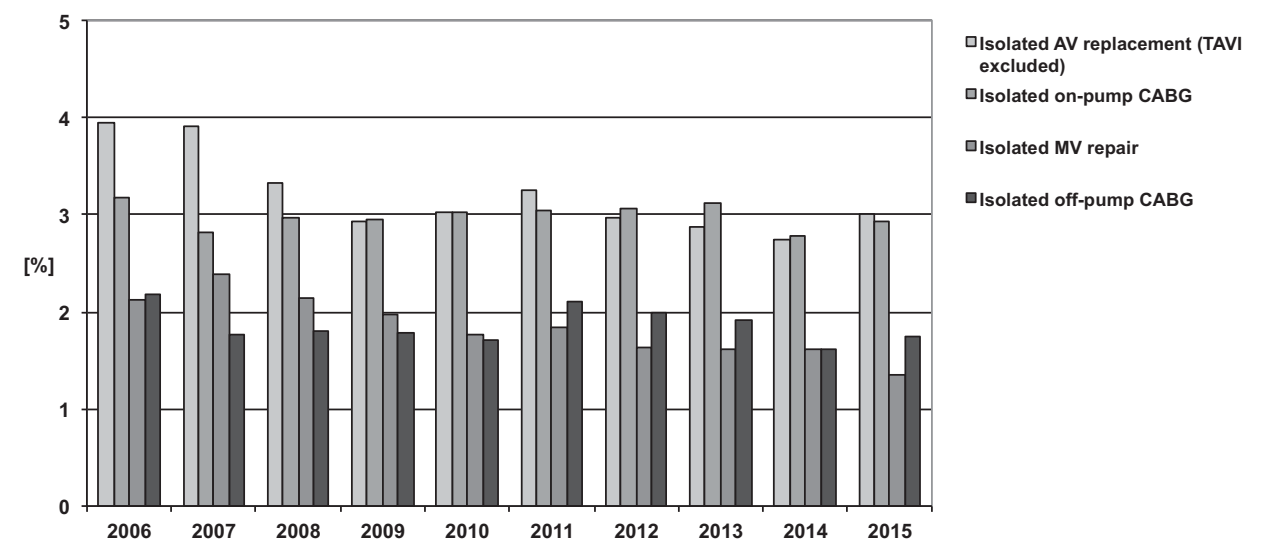

Fig. 2 Unadjusted mortality for selected procedures (2006-2015).

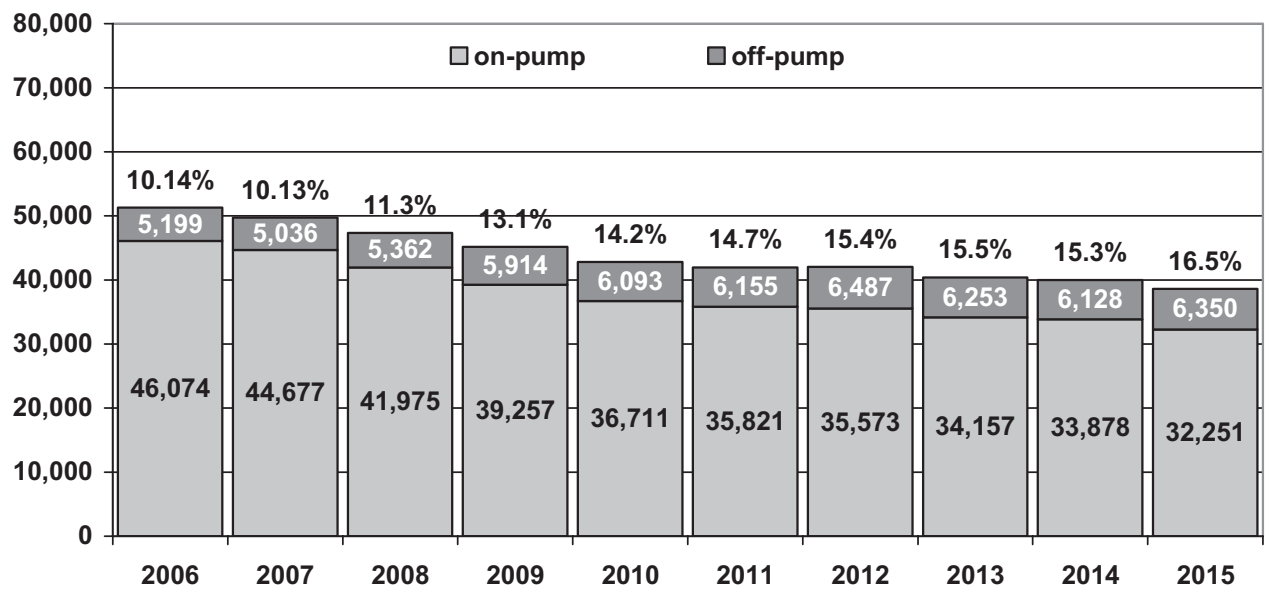

Fig. 3 Isolated coronary artery bypass grafting (2006-2015). 


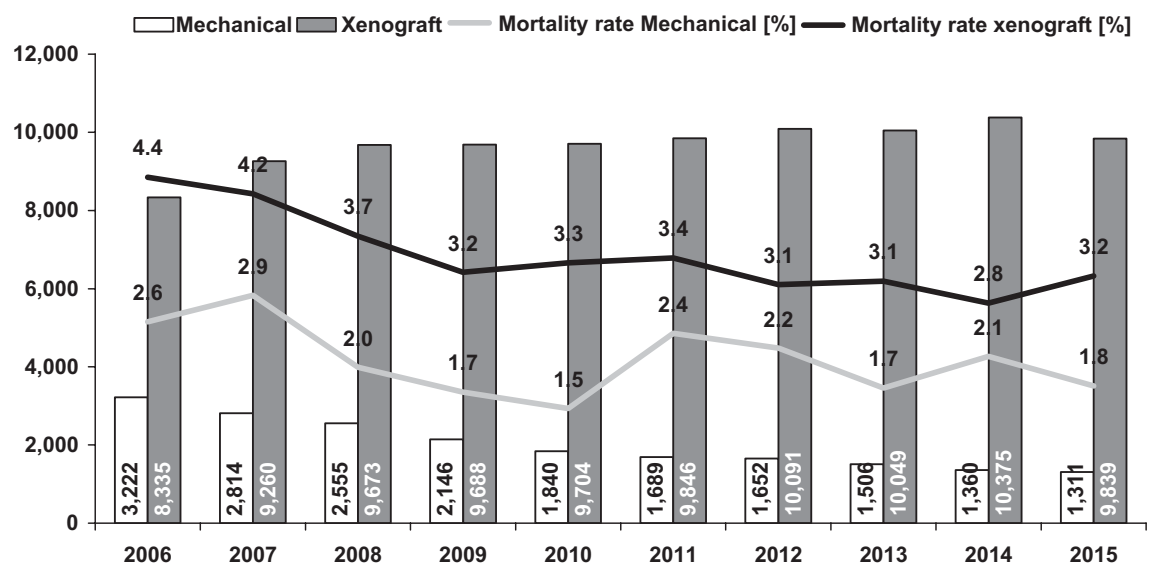

Fig. 4 Isolated aortic valve replacement (2006-2015). Ross procedures, homograft implantations, and transcatheter aortic valve implantation excluded.

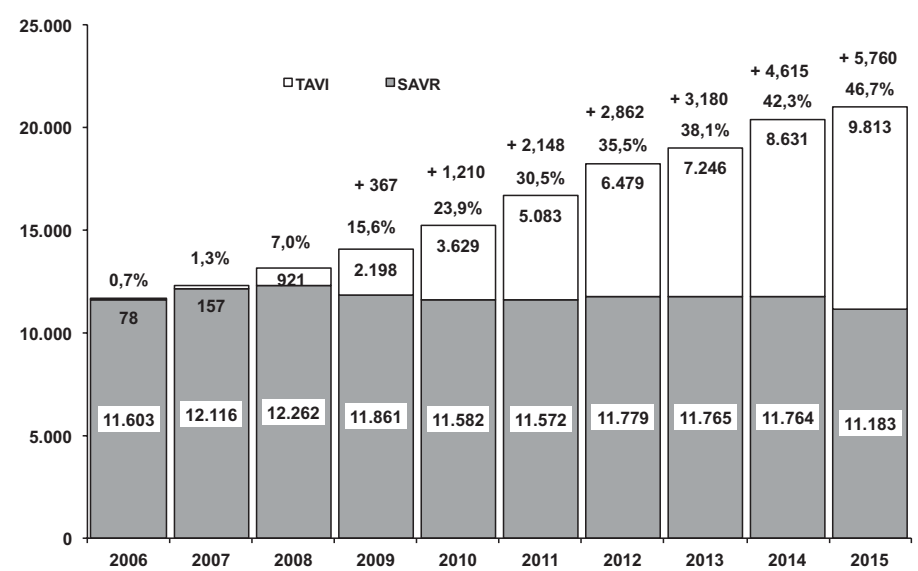

Fig. 5 Isolated aortic valve replacement and transcatheter aortic valve implantation (TAVI). + Additional TAVI procedures calculated from the German legal quality assurance program (§137 SGB V).

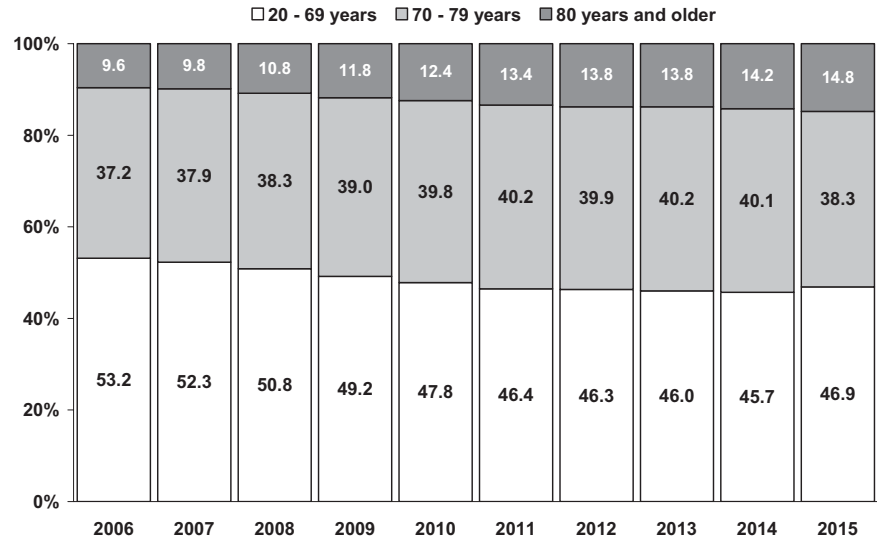

Fig. 6 Age distribution of cardiac procedures (2006-2015). Patients $<20$ years and pacemaker/implantable cardioverter defibrillator procedures excluded. 


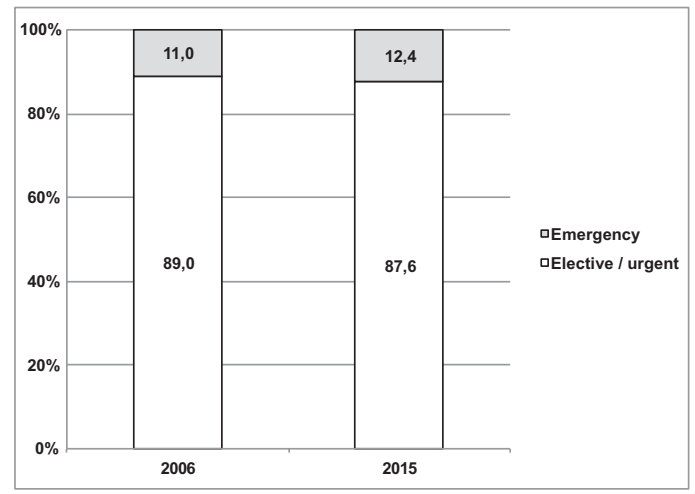

Fig. 7 Urgency categories (2006 vs. 2015).

Emergency $=$ acutely life-threatening cardiac/vascular disease.

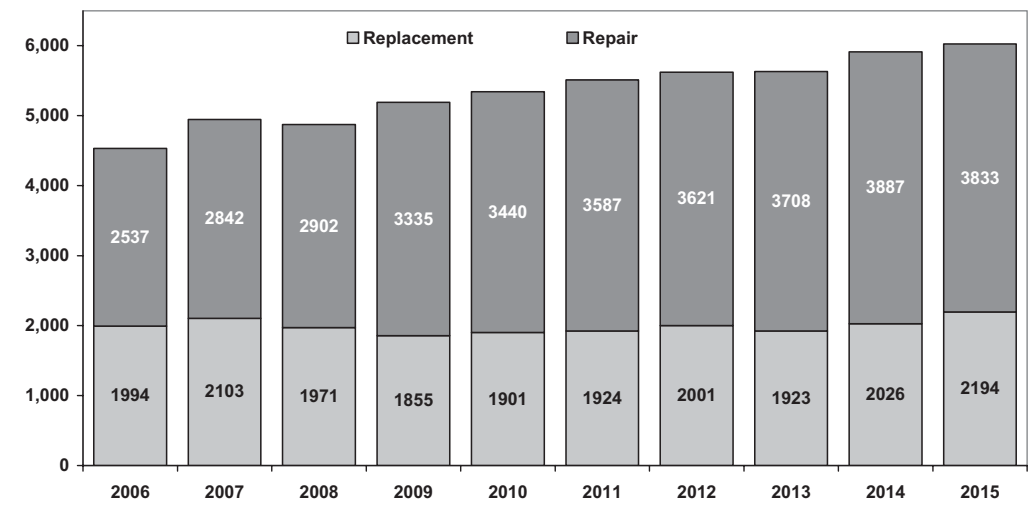

Fig. 8 Isolated mitral valve surgery (2006-2015).

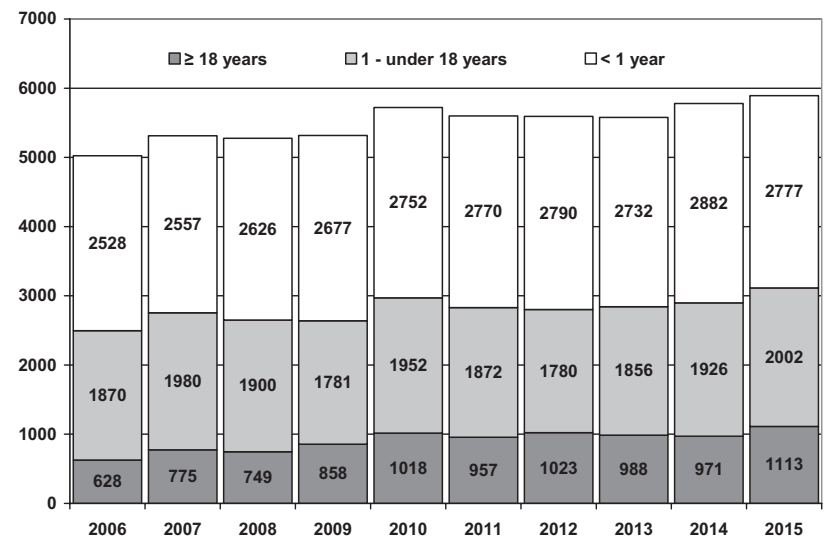

Fig. 9 Age distribution for congenital heart surgery (2006-2015). Bias possible due to the fact that not all relevant procedures can be allocated exactly to the congenital heart disease category in patients older than 18 years (e.g., aortic valve disease).
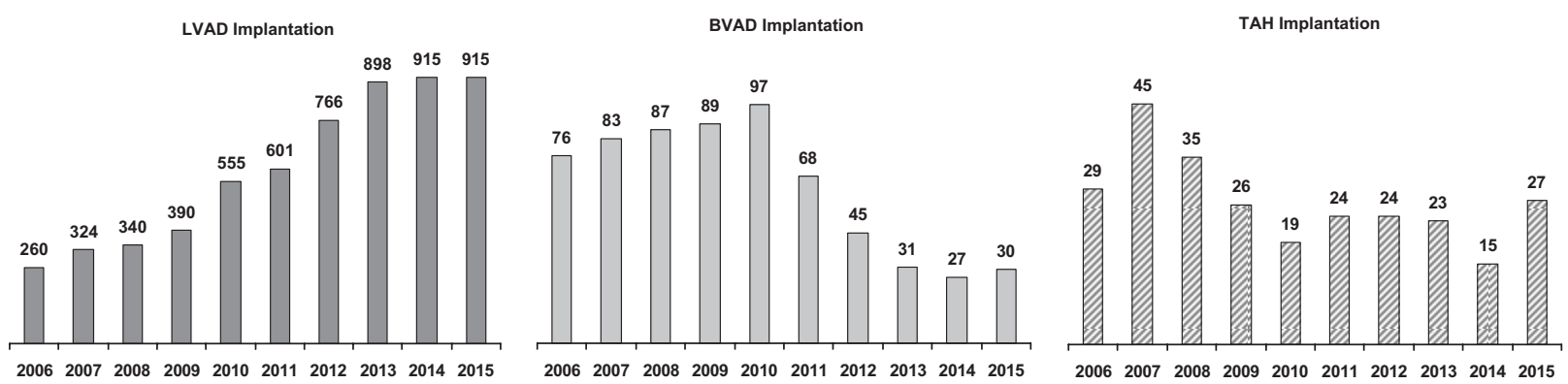

Fig. 10 Development of mechanical circulatory support (2006-2015). 


\section{References}

1 Kalmar P, Irrgang E. Cardiac surgery in the Federal Republic of Germany during 1989. A report by the German Society for Thoracic and Cardiovascular Surgery. Thorac Cardiovasc Surg 1990;38(3):198-200

2 Kalmar P, Irrgang E. Cardiac surgery in the Federal Republic of Germany during 1990. A report by the German Society for Thoracic and Cardiovascular Surgery. Thorac Cardiovasc Surg 1991;39(3):167-169

3 Kalmar P, Irrgang E. Cardiac surgery in Germany during 1991. A report by the German Society for Thoracic and Cardiovascular Surgery. Thorac Cardiovasc Surg 1992;40(3):163-165

4 Kalmar P, Irrgang E. Cardiac surgery in Germany during 1992. A report by the German Society for Thoracic and Cardiovascular Surgery. Thorac Cardiovasc Surg 1993;41(3):202-204

5 Kalmar P, Irrgang E. Cardiac surgery in Germany during 1993. A report by the German Society for Thoracic and Cardiovascular Surgery. Thorac Cardiovasc Surg 1994;42(3):194-196

6 Kalmar P, Irrgang E. Cardiac surgery in Germany during 1994. A report by the German Society for Thoracic and Cardiovascular Surgery. Thorac Cardiovasc Surg 1995;43(3):181-183

7 Kalmar P, Irrgang E. Cardiac surgery in Germany during 1995. A report by the German Society for Thoracic and Cardiovascular Surgery. Thorac Cardiovasc Surg 1996;44(3):161-164

8 Kalmar P, Irrgang E. Cardiac surgery in Germany during 1996. A report by the German Society for Thoracic and Cardiovascular Surgery. Thorac Cardiovasc Surg 1997;45(3):134-137

9 Kalmar P, Irrgang E. Cardiac surgery in Germany during 1997. A report by the German Society for Thoracic and Cardiovascular Surgery. Thorac Cardiovasc Surg 1998;46(5):307-310

10 Kalmar P, Irrgang E. Cardiac surgery in Germany during 1998. A report by the German Society for Thoracic and Cardiovascular Surgery. Thorac Cardiovasc Surg 1999;47(4):260-263

11 Kalmar P, Irrgang E. Cardiac surgery in Germany during 1999. Thorac Cardiovasc Surg 2000;48(4):XXVII-XXX

12 Kalmar P, Irrgang E. Cardiac surgery in Germany during 2000. Thorac Cardiovasc Surg 2001;49(5):33-38

13 Kalmar P, Irrgang E. Cardiac surgery in Germany during 2001: a report by the German Society for Thoracic and Cardiovascular Surgery. Thorac Cardiovasc Surg 2002;50(6):30-35

14 Kalmar P, Irrgang E; German Society for Thoracic and Cardiovascular Surgery. Cardiac surgery in Germany during 2002: a report by German Society for Thoracic and Cardiovascular Surgery. Thorac Cardiovasc Surg 2003;51(5):25-29

15 Kalmar P, Irrgang E; German Society for Thoracic and Cardiovascular Surgery. Cardiac surgery in Germany during 2003: a report by the German Society for Thoracic and Cardiovascular Surgery. Thorac Cardiovasc Surg 2004;52(5):312-317

16 Gummert JF, Funkat A, Krian A. Cardiac surgery in Germany during 2004: a report on behalf of the German Society for Thoracic and Cardiovascular Surgery. Thorac Cardiovasc Surg 2005;53(6): 391-399

17 Gummert JF, Funkat A, Beckmann A, Hekmat K, Ernst M, Krian A. Cardiac surgery in Germany during 2005: a report on behalf of the German Society for Thoracic and Cardiovascular Surgery. Thorac Cardiovasc Surg 2006;54(5):362-371

18 Gummert JF, Funkat A, Beckmann A, et al. Cardiac surgery in Germany during 2006: a report on behalf of the German Society for Thoracic and Cardiovascular Surgery. Thorac Cardiovasc Surg 2007;55(6):343-350

19 Gummert JF, Funkat A, Beckmann A, et al; German Society for Thoracic and Cardiovascular Surgery. Cardiac surgery in Germany during 2007: a report on behalf of the German Society for Thoracic and Cardiovascular Surgery. Thorac Cardiovasc Surg 2008;56(6): 328-336

20 Gummert JF, Funkat A, Beckmann A, et al. Cardiac surgery in Germany during 2008. A report on behalf of the German Society for Thoracic and Cardiovascular Surgery. Thorac Cardiovasc Surg 2009;57(6):315-323

21 Gummert JF, Funkat A, Beckmann A, et al. Cardiac surgery in Germany during 2009. A report on behalf of the German Society for Thoracic and Cardiovascular Surgery. Thorac Cardiovasc Surg 2010;58(7):379-386

22 Gummert JF, Funkat AK, Beckmann A, et al. Cardiac surgery in Germany during 2010: a report on behalf of the German Society for Thoracic and Cardiovascular Surgery. Thorac Cardiovasc Surg 2011;59(5):259-267

23 Funkat AK, Beckmann A, Lewandowski J, et al. Cardiac surgery in Germany during 2011: a report on behalf of the German Society for Thoracic and Cardiovascular Surgery. Thorac Cardiovasc Surg 2012;60(6):371-382

24 Beckmann A, Funkat AK, Lewandowski J, et al. Cardiac surgery in Germany during 2012: a report on behalf of the German Society for Thoracic and Cardiovascular Surgery. Thorac Cardiovasc Surg 2014;62(1):5-17

25 Funkat A, Beckmann A, Lewandowski J, et al. Cardiac surgery in Germany during 2013: a report on behalf of the German Society for Thoracic and Cardiovascular Surgery. Thorac Cardiovasc Surg 2014;62(5):380-392

26 Beckmann A, Funkat AK, Lewandowski J, et al. Cardiac Surgery in Germany during 2014: a report on behalf of the German Society for Thoracic and Cardiovascular Surgery. Thorac Cardiovasc Surg 2015;63(4):258-269

27 Gammie JS, Zhao Y, Peterson ED, O’Brien SM, Rankin JS, Griffith BPJ. J. Maxwell Chamberlain Memorial Paper for adult cardiac surgery. Less-invasive mitral valve operations: trends and outcomes from the Society of Thoracic Surgeons Adult Cardiac Surgery Database. Ann Thorac Surg 2010;90(5):1401-1408, 1410.e1, discussion 1408-1410

28 Vahanian A, Alfieri O, Andreotti F, et al; Joint Task Force on the Management of Valvular Heart Disease of the European Society of Cardiology (ESC); European Association for Cardio-Thoracic Surgery (EACTS). Guidelines on the management of valvular heart disease (version 2012). Eur Heart J 2012;33(19):2451-2496

29 Beckmann A, Hamm C, Figulla HR, et al; GARY Executive Board. The German Aortic Valve Registry (GARY): a nationwide registry for patients undergoing invasive therapy for severe aortic valve stenosis. Thorac Cardiovasc Surg 2012;60(5):319-325

30 Hamm CW, Möllmann H, Holzhey D, et al; GARY-Executive Board. The German Aortic Valve Registry (GARY): in-hospital outcome. Eur Heart J 2014;35(24):1588-1598

31 Mohr FW, Holzhey D, Möllmann H, et al; GARY Executive Board. The German Aortic Valve Registry: 1-year results from 13,680 patients with aortic valve disease. Eur J Cardiothorac Surg 2014; 46(5):808-816

32 Lauten A, Figulla HR, Möllmann H, et al; GARY Executive Board. TAVI for low-flow, low-gradient severe aortic stenosis with preserved or reduced ejection fraction: a subgroup analysis from the German Aortic Valve Registry (GARY). EuroIntervention 2014;10(7):850-859

33 Walther T, Hamm C, Schuler G, et al. ; GARY Executive Board. Perioperative results and complications in 15,964 transcatheter aortic valve replacements: prospective data from the GARY Registry. J Am Coll Cardiol 2015;65(20):2173-2180

34 Herbert MA, Prince SL, Williams JL, Magee MJ, Mack MJ. Are unaudited records from an outcomes registry database accurate? Ann Thorac Surg 2004;77(6):1960-1964, discussion 1964-1965 